AN. MED. InTERNa (Madrid) Vol. 18, N. ${ }^{\circ} 4$, pp. 191-194, 2001

\title{
Arteritis de células gigantes y polimialgia reumática: diagnóstico y evolución de 90 casos
}

\author{
A. RIVERO PUENTE, J.I. BERASATEGUI CALDERÓN, J.M. MURIE CARRILLO DE \\ ALBORNOZ, M. RIVERO MARCOTEGUI, F. GARCÍA-BRAGADO ACÍN
}

Servicios de Medicina Interna y Anatomía Patológica. Hospital Virgen del Camino. Pamplona

\author{
GIANT CELL ARTERITIS AND POLYMYALGIA RHEUMATICA: \\ DIAGNOSIS AND EVOLUTION OF 90 CASES
}

\begin{abstract}
RESUMEN
Objetivo: Comparar el cuadro clínico de la arteritis de células gigantes (ACG) y el de la polimialgia reumática (PMR). Valorar los criterios diagnósticos del American College of Rheumatology contrastándolos con nuestros resultados.

Pacientes y métodos: Estudio retrospectivo de 90 enfermos diagnosticados de ACG ( $\mathrm{n}=57,55$ diagnosticados por biopsia) y de PMR $(\mathrm{n}=$ 33) en los últimos 10 años.

Resultados: Presentaron cefalea 45 enfermos $(78,94 \%)$ con ACG y 7 $(21,21 \%)$ con PMR $(\mathrm{p}<0,001)$, síndrome polimiálgico $15(26,31 \%)$ con ACG y $33(100 \%)$ con PMR ( $<0,001)$, claudicación mandibular o lingual $14(24,57 \%)$ con ACG y $2(6,06 \%)$ con PMR $(\mathrm{p}<0,05)$ y alteraciones visuales $9(15,79 \%)$ con ACG. La VSG fue $\geq 50 \mathrm{~mm} / \mathrm{h}$ en 84 enfermos $(93,33 \%)$ y $\geq 100 \mathrm{~mm} / \mathrm{h}$ en 43 de ellos $(51,19 \%)$. La VSG se normalizó (£ $20 \mathrm{~mm} / \mathrm{h}$ ) en menos de 8 semanas en 64 enfermos $(76,19 \%)$. En el momento del diagnóstico 61 enfermos $(61,77 \%)$ presentaron anemia, 17 de ellos $(27,86 \%)$ de grado severo $(\mathrm{Hb}<10 \mathrm{~g} / \mathrm{dl})$. Tras tratamiento esteroideo se recuperaron de la anemia 43 enfermos $(70,49 \%)$, todos en menos de 12 semanas y $25(58,13 \%)$ en menos de 8 semanas.

Conclusiones: Los síntomas cefálicos orientaron el diagnóstico en la ACG y son predictivos de biopsia de arteria temporal positiva. La anemia y su rápida normalización tras tratamiento exclusivo con glucocorticoides son datos que pueden ayudar en el diagnóstico de estas entidades.
\end{abstract}

PALABRAS CLAVE: Arteritis de células gigantes. Polimialgia reumática. Anemia.

\begin{abstract}
Objective: To compare the clinical features of giant cell arteritis $(G C A)$ and polymyalgia rheumatic (PMR), and to evaluate the criteria proposed by the American College of Rheumatology in our results.

Patients and methods: A retrospective analysis of 90 patients with GCA $(n=57,55$ of whom were diagnosed by biopsy) and PMR $(n=33)$ diagnosed over the last 10 years.

Results: Headache was present in 45 patients (78.94\%) with GCA and in $7(21.21 \%)$ with PMR ( $p<0.001)$; polymyalgic syndrome was observed in 15 patients (26.31\%) with GCA and in $33(100 \%)$ with PMR $(p<0.001)$; jaw or tongue claudication was observed in 14 patients $(24.57 \%)$ with $G C A$ and in $2(6.06 \%)$ with PMR $(p<0.05)$, and visual disturbances were only present in 9 patients (15.79\%) with GCA. The erythrocyte sedimentation rate (ESR) was $\geq 50 \mathrm{~mm} / \mathrm{h}$ in 84 patients (93.33\%), and $\geq 100 \mathrm{~mm} / \mathrm{h}$ in 43 of them (51.19\%). The ESR became normal ( $£ 20 \mathrm{~mm} / \mathrm{h})$ in less of 8 weeks after the treatment was started in 64 patients $(76.19 \%)$. At the time of diagnosis, 61 patients $(61.67 \%)$ had anemia, which was severe $(\mathrm{Hb}<10 \mathrm{~g} / \mathrm{dl})$ in 17 cases $(27.86 \%)$. After ste roid treatment 43 patients $(70.49 \%)$ improved their anemia in less of 12 weeks, and 25 of them (58.13\%) in less of 8 weeks.

Conclusions: The cranial symptoms were predictive for a positive temporal artery biopsy. The anemia and its quick normalitation after ste roid treatment can help to the diagnostic.
\end{abstract}

KEY WORDS: Giant cell arteritis. Polymyalgia rheumatic. Anemia.

Rivero Puente A, Berasategui Calderón J.I, Murie Carrillo de Albornoz J.M, Rivero Marcotegui M, García-Bragado Acín F. Arteritis de células gigantes y polimialgia reumática: diagnóstico y evolución de 90 casos. An Med Interna (Madrid) 2001; 18: 191-194.

\section{INTRODUCCIÓN}

La arteritis de células gigantes (ACG) es la vasculitis más frecuente en la edad avanzada (1-3). Los criterios que orientan el diagnóstico de ACG son fundamentalmente clínicos, aunque el diagnóstico definitivo requiere la demostración de arteritis en la biopsia de la arteria temporal (AT). Sus criterios de clasificación siguen siendo los propuestos por el American
College of Rheumatology (ACR) en 1990 (4). La polimialgia reumática (PMR) es un síndrome clínico, que afecta también a personas mayores, caracterizado por dolor y rigidez en cintura escapular, pélvica y cuello (5). A falta de unos criterios diagnósticos multicéntricos y estandarizados, unos de los utilizados con mayor frecuencia en la práctica clínica para la PMR son los definidos por Healey en la Mason Clinic que datan de 1984 (6).

Trabajo aceptado: 11 de Septiembre de 2000

Correspondencia: A. Rivero Puente. Servicio de Medicina Interna. Hospital Virgen del Camino. C/ Irunlarrea, 4. 31008 Pamplona. 
La relación que existe entre ambos procesos no está todavía aclarada, pero va prevaleciendo la hipótesis, defendida especialmente por los autores escandinavos $(2,7)$, de que se trata de una misma entidad patológica con diferente expresión clínica. De hecho, aproximadamente la mitad de los pacientes con ACG presenta un síndrome clínico de PMR (3) que incluso puede ser la única manifestación de la enfermedad (7); más aún, en estudios recientes se han encontrado signos histológicos de arteritis en la biopsia de la AT hasta en un 15\% de los pacientes con PMR sin síntomas cefálicos de vasculitis $(1,8)$.

Hemos revisado nuestra casuística con el propósito de comparar el cuadro clínico de la ACG y de la PMR, estudiar la rentabilidad diagnóstica de la biopsia de la AT y valorar los criterios del ACR para la ACG y de Healey para la PMR contrastándolos con nuestros resultados.

\section{PACIENTES Y MÉTODOS}

Estudio retrospectivo de todos los enfermos diagnosticados de ACG y de PMR en nuestro hospital durante los últimos 10 años (Enero de 1989 Diciembre de 1998). La identificación de los casos se llevó a cabo consultando la base de datos de los Servicios de Medicina Interna y Anatomía Patológica.

\section{CRITERIOS DIAGNÓSTICOS}

Diagnóstico de ACG. Se estableció cuando la biopsia de la AT era positiva (infiltrado inflamatorio por células mononucleares en la pared arterial y fragmentación de la lámina elástica interna, con o sin células gigantes multinucleadas). Excepcionalmente se consideró el diagnóstico en pacientes con biopsia de la AT negativa si reunían al menos 3 de los criterios clínicos propuestos por el ACR (4): a) edad $\geq 50$ años; b) VSG $\geq 50 \mathrm{~mm} / \mathrm{h}$; c) cefalea de aparición reciente; d) anomalía/s en la exploración de la AT.

Diagnóstico de PMR. Se siguieron los criterios propuestos por Healey (6): a) síndrome de polimialgia reumática: dolor persistente (durante al menos un mes) que afecta como mínimo a dos de las siguientes zonas: cuello, cintura escapular y cintura pélvica; b) rigidez matutina durante $>1 \mathrm{~h} ; \mathrm{c}$ ) edad $\geq 50$ años; d) VSG $\geq 40 \mathrm{~mm} / \mathrm{h}$; e) respuesta rápida al tratamiento con glucocorticoides; f) ausencia de otras enfermedades osteomusculares definidas.

\section{DEFINICIONES}

Las anomalías en la exploración de la AT incluyeron dolor, hiperestesia, inflamación local y/o alteraciones del pulso. Se consideraron como alteraciones visuales la pérdida brusca de visión uní o bilateral y los defectos campimétricos. La pérdida de peso se consideró significativa si era $>3 \mathrm{Kg}$ en tres meses. Se consideró que existía anemia cuando la hemoglobina $(\mathrm{Hb})$ era $<13 \mathrm{~g} / \mathrm{dl}$ en varones y $<12 \mathrm{~g} / \mathrm{dl}$ en mujeres. Se definió como normalización de la VSG cuando era $£ 20$ $\mathrm{mm} / \mathrm{h}$. La curación de la enfermedad se definió como la ausencia total de sintomatología clínica junto con normalización de la VSG y como recidiva se consideró la reaparición de los síntomas clínicos y de la elevación de la VSG.

\section{ESTUDIO ESTADÍSTICO}

Los datos del estudio se introdujeron y analizaron en el paquete informático SPSS. Se analizó en primer lugar la distribución de las poblaciones de interés mediante la aplicación de la prueba de Shapiro-Wilks y el cálculo de los coeficientes de sesgo y curtosis. Al no ser poblaciones que siguieran una distribución normal se realizó el cálculo de la mediana y de los fractiles $0,025\left(\mathrm{~F}_{0,025}\right)$ y $0,975\left(\mathrm{~F}_{0,975}\right)$ para definir cada una de las poblaciones de interés. Se aplicaron las pruebas de MannWhitney y Wilconson para el estudio comparativo poblacional. Se realizaron tablas de contingencia calculando la $\chi^{2}$ y la $\mathrm{p}$ de Fisher cuando se trataron de variables cualitativas.

\section{RESULTADOS}

Durante los últimos 10 años, 13.548 enfermos de edad $\geq$ a los 50 años han sido hospitalizados en nuestro servicio. Se incluyen en este estudio 90 enfermos: 57 diagnosticados de ACG y 33 diagnosticados de PMR. Esto representa una incidencia para la ACG de 4,2 casos por 1.000 pacientes hospitalizados de edad $\geq$ a los 50 años y de 2,4 casos para la PMR. En la Tabla I se recogen los datos demográficos de la población estudiada.

\begin{tabular}{llll}
\multicolumn{4}{c}{ TABLA I } \\
\multicolumn{4}{c}{ DATOS DEM O GRÁFICOS } \\
\hline & $\begin{array}{l}\text { ACG } \\
(n=57)\end{array}$ & $\begin{array}{l}\text { PM R } \\
(\mathrm{n}=33)\end{array}$ & $\begin{array}{l}\text { Total } \\
(\mathrm{n}=90)\end{array}$ \\
\hline $\begin{array}{llll}\text { Edad } \\
\text { X }\end{array}$ & 72,70 & 72,78 & 72,73 \\
De & 7,07 & 8,08 & 7,41 \\
Rango & $56-85$ & $52-86$ & $52-86$ \\
Sexo & & & \\
M ujeres & 30 & 17 & 47 \\
Varones & 27 & 16 & 43 \\
Relación M /V & 1,115 & 1,06 & 1,09 \\
\hline
\end{tabular}

Se representa el valor medio (x), la desviación estándar (De) y la relación mujer/varón (M/V).

La biopsia de la AT fue positiva en 55 de los 57 enfermos diagnosticados de ACG. Todos ellos presentaron además al menos 3 de los criterios diagnósticos del ACR. Los 2 enfermos con biopsia negativa se diagnosticaron de ACG por reunir al menos 3 de los criterios clínicos descritos. La longitud media de la biopsia de la AT fue de 1,1 cm (rango: 0,5-2). Se practicó biopsia de la AT en 27 de los 33 pacientes con PMR siendo su estudio histológico negativo en todos ellos (en un caso la biopsia fue bilateral). Uno de estos enfermos era mujer de 70 años con clínica muy sugestiva de PMR referida especialmente a cinturas pélvicas y zonas gemelares, y aunque la biopsia de la AT fue negativa, una biopsia del músculo cuádriceps mostró signos de arteritis con células gigantes en una arteria de mediano calibre.

Globalmente, las manifestaciones clínicas más frecuentes fueron cefalea (en el $57,77 \%$ de los casos), síndrome de polimialgia reumática $(53,33 \%)$, pérdida de peso $(36,66 \%)$, fiebre 
$(32,22 \%)$, claudicación mandibular y/o lingual (17,77\%), artromialgias $(17,77 \%)$ y alteraciones visuales $(10 \%)$. En la Tabla II se exponen comparativamente las manifestaciones clínicas del grupo de pacientes con ACG y con PMR.

TABLA II

\begin{tabular}{|c|c|c|c|c|c|}
\hline \multicolumn{6}{|c|}{$\begin{array}{l}\text { M ANIFESTACIO NES CLÍNICAS. COM PARACIÓ N } \\
\text { ENTRE LA ACG Y LA PM R }\end{array}$} \\
\hline & \multicolumn{2}{|c|}{ ACG $(n=57)$} & \multicolumn{2}{|c|}{ PMR $(n=33)$} & \multirow[t]{2}{*}{$p\left(\chi^{2}\right)$} \\
\hline & $n$ & $\%$ & $n$ & $\%$ & \\
\hline Cefalea & 45 & 78,94 & 7 & 21,21 & $<0,001$ \\
\hline PM R & 15 & 26,3 & 33 & 100 & $<0,001$ \\
\hline Pérdida de peso & 22 & 38,59 & 11 & 33,33 & NS \\
\hline Fiebre & 19 & 33,33 & 10 & 30,30 & NS \\
\hline $\begin{array}{l}\text { Claudicación mandibular } \\
\text { y/ o lingual }\end{array}$ & 14 & 24,57 & 2 & 6,06 & $<0,05$ \\
\hline M ialgias y artralgias & 6 & 10,52 & 10 & 30,30 & $<0,05$ \\
\hline Alteraciones visuales & 9 & 15,79 & 0 & 0,00 & - \\
\hline
\end{tabular}

Se representan los valores de $p$ de Fisher obtenidos tras la realización de la prueba de la $\chi^{2}$. NS: valor de $p$ sin significación estadística.

El valor medio de la VSG en el momento del diagnóstico fue de $107 \mathrm{~mm} / \mathrm{h}$ en los pacientes con ACG y de $91 \mathrm{~mm} / \mathrm{h}$ en los pacientes con PMR ( $\mathrm{p}=0,05)$ (Tabla III). En 56 de los 57 enfermos con ACG $(98,24 \%)$ la VSG fue $\geq 50 \mathrm{~mm} / \mathrm{h}$, y de ellos en $25(44,64 \%) \geq 100 \mathrm{~mm} / \mathrm{h}$. En el grupo de pacientes con PMR la VSG fue $\geq 50 \mathrm{~mm} / \mathrm{h}$ en $28 \operatorname{casos}(84,84 \%)$ y $\geq$ $100 \mathrm{~mm} / \mathrm{h}$ en 18 de ellos (64,28\%). Globalmente, 84 de los 90 enfermos estudiados $(93,33 \%)$ presentaron una VSG inicial $\geq$ $50 \mathrm{~mm} / \mathrm{h}$, siendo $\geq 100 \mathrm{~mm} / \mathrm{h}$ en 43 de ellos $(51,19 \%)$. Tras iniciar tratamiento con glucocorticoides, la VSG se normalizó en menos de 8 semanas en 64 enfermos $(76,19 \%)$.

\section{TABLA III}

DATOS ANALÍTICOS EN EL MOM ENTO DEL DIAGNÓSTICO

\begin{tabular}{|c|c|c|c|c|c|}
\hline & \multicolumn{2}{|c|}{$A C G(n=57)$} & \multicolumn{2}{|c|}{ PMR $(n=33)$} & \multirow[t]{2}{*}{$p$} \\
\hline & Mediana & $\mathrm{F}_{0,025}-\mathrm{F}_{0,975}$ & Mediana & $\mathrm{F}_{0,025}-\mathrm{F}_{0,975}$ & \\
\hline $\mathrm{VSG}_{1}(\mathrm{~mm} / \mathrm{h})$ & 107 & $54-138$ & 91 & $4,45-139$ & 0,05 \\
\hline $\mathrm{Hb}_{1}(\mathrm{~g} / \mathrm{dl})$ & 11 & $9,5-15$ & 11,8 & $7,57-15,53$ & 0,01 \\
\hline $\mathrm{Hcrto}_{1}(\%)$ & 32,7 & $28-41,4$ & 35,3 & $22,75-48,52$ & $<0,01$ \\
\hline
\end{tabular}

$\mathrm{Hb1}$ : hemoglobina en el momento del diagnóstico; Hcrto1: hematocrito en el momento del diagnóstico; VSG1: VSG en el momento del diagnóstico. Se representa la $p$ obtenida tras la aplicación de la prueba $U$ de $M$ ann-W hitney-W ilconson.

El valor medio de la $\mathrm{Hb}$ en el momento del diagnóstico fue de $11 \mathrm{~g} / \mathrm{dl}$ en el grupo de ACG y de 11,8 g/dl en el grupo de PMR $(\mathrm{p}=0,01)$ (Tabla III). De los 90 enfermos estudiados, $61(67,77 \%$ ) (30 varones y 31 mujeres) presentaron anemia en el momento del diagnóstico, y en $17(27,86 \%)$ el grado inicial de la misma fue intenso con cifras de $\mathrm{Hb} £ 10$ g/dl. Las características de la anemia aparecen en la Tabla IV. Tras el inicio del tratamiento con glucocorticoides, la cifra de $\mathrm{Hb}$ se normalizó en 43 enfermos (70,49\%), todos en menos de 12 semanas y 25 de ellos $(58,13 \%)$ en menos de 8 semanas. No se apreciaron diferencias significativas en la recuperación de la anemia ni entre los dos grupos diagnósticos ni entre los diferentes grados iniciales de anemia. En la Tabla V se recogen los valores medios de VSG, Hb y hematocrito antes y después del tratamiento esteroideo.

TABLA IV

CARACTERÍSTICAS DE LA ANEM IA

\begin{tabular}{lll}
\hline & M ediana & $\mathrm{F}_{0,025}-\mathrm{F}_{0,975}$ \\
\hline VCM (fl) & 86,5 & $79,8-101$ \\
Reticulocitos (o/ oo) & 6 & $5-13$ \\
Hierro $(\mu \mathrm{g} / \mathrm{dl})$ & 25 & $15-59$ \\
Ferritina $(\mathrm{ng} / \mathrm{ml})$ & 266 & $11-597$ \\
Transferrina $(\mathrm{mg} / \mathrm{dl})$ & 174 & $88-252$ \\
\hline
\end{tabular}

TABLA V

DIFERENCIAS ANALÍTICAS ENTRE EL MOM ENTO DEL DIAGNÓ STICO Y TRAS EL TRATAM IENTO

\begin{tabular}{lllllll}
\hline & \multicolumn{3}{c}{ ACG $(n=57)$} & \multicolumn{2}{c}{$\operatorname{PMR}(n=33)$} \\
& Mediana & $\mathrm{F}_{0,025}-\mathrm{F}_{0,975}$ & $\mathrm{p}$ & Mediana & $\mathrm{F}_{0,025}-\mathrm{F}_{0,975}$ & $\mathrm{p}$ \\
\hline $\mathrm{VSG}_{1}$ & 107 & $54-138$ & 0 & 91 & $4,45-139$ & $<0,001$ \\
VSG $_{2}$ & 12 & $2-51$ & & 11 & $2-98,37$ & \\
$\mathrm{Hb}_{1}$ & 11 & $9,5-15$ & 0 & 11,8 & $7,87-15,53$ & $<0,001$ \\
$\mathrm{Hb}_{2}$ & 13,9 & $10,5-16,6$ & & 14,0 & $10,4-16,6$ & \\
$\mathrm{Hcrto}_{1}$ & 32,7 & $28-41,4$ & 0 & 35,3 & $22,75-48,52$ & $<0,001$ \\
Hcrto $_{2}$ & 40,5 & $31,9-45,5$ & & 41 & $29,39-51,56$ & \\
\hline
\end{tabular}

$\mathrm{VSG}_{1}$ : VSG en el momento del diagnóstico $(\mathrm{mm} / \mathrm{h}) ; \mathrm{VSG}_{2}$ : VSG tras tratamiento; $\mathrm{Hb}_{1}$ : Hemoglobina en el momento del diagnóstico ( $\left.\mathrm{g} / \mathrm{dl}\right) ; \mathrm{Hb}_{2}$ : Hemoglobina tras tratamiento; $\mathrm{Hcrto}_{1}$ : Hematocrito en el momento del diagnóstico (\%); $\mathrm{Hcrto}_{2}$ : Hematocrito tras tratamiento. Se representa la $\mathrm{p}$ obtenida tras la aplicación de la prueba U de M ann-W hitney-W ilconson.

La concentración sérica de fosfatasa alcalina, determinada en el momento del diagnóstico en 68 casos, estaba ligeramente elevada en $11(16,17 \%)$.

En el grupo de pacientes con ACG la dosis media de inicio de prednisona fue de $50 \mathrm{mg}$ diarios y la duración del tratamiento de 18 meses, mientras que en los pacientes con PMR la dosis diaria media de inicio de prednisona fue de $30 \mathrm{mg}$ y la duración del tratamiento de 20 meses.

El tiempo medio de seguimiento fue de 3,41 años. Un total de 14 enfermos presentaron recaídas al disminuir la dosis de prednisona: 9 de los enfermos con ACG y 5 con PMR. En este período fallecieron 7 de los enfermos con ACG y 3 con PMR, todos por causas aparentemente no relacionadas con la enfermedad de base.

\section{DISCUSIÓN}

Los criterios que orientan el diagnóstico de ACG son fundamentalmente clínicos, pero el diagnóstico de certeza sólo puede obtenerse mediante la confirmación histológica. En 1990 el ACR (4) propuso unos criterios que, sin pretender sustituir como herramienta diagnóstica a la biopsia de la AT, pudieran ayudar a uniformar su diagnóstico. Según estos cri- 
terios, el $100 \%$ de los pacientes de nuestra serie podían clasificarse como ACG. Por otra parte, y debido a que las lesiones histológicas de la ACG son focales, una biopsia normal no excluye completamente el diagnóstico. Por ello en casi todas las series $(3,4)$ se incluyen casos con biopsia normal. La longitud media de las arterias biopsiadas en nuestro estudio fue sólo de $1,1 \mathrm{~cm}$. Longitud que es menor de los 3-4 cm que propugnan algunos autores (8).

La etiopatogenia de la PMR y su relación con la ACG no está del todo aclarada. Para algunos $(9,10)$ son entidades bien diferenciadas y defienden que las estructuras afectadas en la PMR son la articulación proximal, la vaina tendinosa y la membrana sinovial. Sin embargo, las muestras positivas de biopsia de la AT en pacientes con PMR que no presentan síntomas craneales ha llevado a otros $(2,4,7,11)$ a considerar que la vasculitis es la responsable de los síntomas osteomusculares de la PMR. En nuestra serie, en todos los pacientes con PMR y ausencia de síntomas y signos cefálicos, en los que se practicó biopsia de la AT ésta fue negativa, por lo que no nos parece que esté justificado la práctica de biopsia de la AT en los cuadros de PMR sin manifestaciones cefálicas, criterio defendido también por otros autores $(12,13)$. No obstante, el caso descrito de la enferma con una biopsia muscular que mostró signos de arteritis nos parece que es demostrativo de la naturaleza vascular del síndrome.

En nuestra serie la clínica fue similar a otras $(3,14)$, siendo la cefalea, las alteraciones visuales y la claudicación mandibular significativamente más frecuentes en la ACG y las artro- mialgias en la PMR. El 26,3\% de los pacientes con ACG presentaba síndrome polimiálgico.

Una VSG muy alta es característica de ambas entidades, constituyendo uno de sus criterios diagnósticos $(4,6)$. No obstante, se han comunicado casos de ACG y de PMR con VSG normal con cifras de hasta el $15 \%$ y el $20 \%$ respectivamente (15-18). En nuestro estudio, sólo un enfermo con ACG presentaba una VSG $<50 \mathrm{~mm} / \mathrm{h}$ y sólo 3 con PMR tenían una $\mathrm{VSG}<40 \mathrm{~mm} / \mathrm{h}$. A nuestro juicio debemos ser muy exigentes y restrictivos en el diagnóstico de ACG y/o PMR sin elevación significativa de la VSG.

El $67,7 \%$ de nuestros pacientes presentaba anemia, en algunos casos de grado intenso. Se trataba de una anemia de proceso crónico (excepto en una paciente en que fue una anemia megaloblástica por déficit de B12) bien tolerada que se recuperó en un breve período de tiempo a pesar de la avanzada edad de los enfermos. Si bien en la literatura se ha hecho referencia a la anemia, incluso en ocasiones intensa, como manifestación de esta enfermedad (19-23) y, recientemente, se ha observado que su ausencia puede ser un predictor de complicaciones visuales (24), a raíz de nuestros resultados queremos hacer énfasis en su elevada frecuencia, su intensidad y su rápida normalización tras el tratamiento exclusivo con glucocorticoides.

En nuestra casuística se confirma la alta sensibilidad de los criterios propuestos por el ACR para la clasificación de la ACG.

\section{Bibliografía}

1. Salvarani C, Gabriel SE, O’Fallon WM, Hunder GG. Epidemiology of polymyalgia rheumatica in Olmsted County, Minnesota, 1970-1991. Arthritis Rheum 1995; 38: 369-373.

2. Bengtsson BA, Malmvall BE. The epidemiology of giant cell arteritis including temporal arteritis and polymyalgia rheumatica: incidences of different clinical presentations and eye complications. Arthritis Rheum 1981; 24: 899-904.

3. Baldursson O, Steinsson K, Bjornsson J, Lie JT. Giant cell arteritis in Iceland. An epidemiologic and histopathologic analysis. Arthritis Rheum 1994; 37: 1007-1012.

4. Hunder GG, Bloch DA, Michel BA, Stevens MB, Arend WP, Calabrese LH et al. The American College of Rheumatology 1990 criteria for the classification of giant cell arteritis. Arthritis Rheum 1990; 33: 1122-1128.

5. Calvo JM, Ramos JL, Pérez M. Polimialgia reumática. An Med Interna (Madrid) 2000; 17: 42-46.

6. Healey LA. Long-term follow-up of polymyalgia rheumatica: evidence for sinovitis. Semin Arthritis Rheum 1984; 13: 322-328.

7. Boesen P, Sorensen SF. Giant cell arteritis, temporal arteritis, and polymyalgia rheumatic in a Danish county. Arthritis Rheum 1987; 30: 294-299.

8. Hunder GG. Giant cell arteritis and polymyalgia rheumatica. Med Clin North Am 1997; 81: 195-219.

9. Salvarani C, Gabriel S, Hunder GG. Distal extremity swelling with pitting edema in polymyalgia rheumatica. Report of nineteen cases. Arthritis Rheum 1996; 39: 73-80.

10. Olivieri I, Salvarani C, Cantini F. Remitting distal extremity swelling with pitting edema: a distinct syndrome or a clinical feature of different inflammatory rheumatic diseases?. J Rheumatol 1997; 24: 249-252.

11. Blockmans D, Maes A, Stroobants S, Nuyts J, Bormans G, Knockaert $D$ et al. New arguments for a vasculitic nature of polymyalgia rheumatica using positron emission tomography. Rheumatology 1999; 38 : 444-447.

12. Salvarani C, Macchioni P, Boiardi L. Polimialgia reumática. Lancet (Ed. esp) 1997; 350: 43-47.

13. Rodríguez-Valverde V, Sarabia JM, González-Gay MA, Figueroa M,

Armona J, Blanco R et al. Risk factors and predictive models of giant cell arteritis in polymyalgia rheumatica. Am J Med 1997; 102: 331-336.

14. Armona J, Rodríguez-Valverde V, González-Gay MA, Figueroa M, Fernández-Sueiro JL, Blanco R et al. Arteritis de células gigantes. Estudio de 191 pacientes. Med Clin (Barc) 1995; 105: 734-737.

15. Brittain GPH, McIlwaine GG, Bell JA, Gibson JM. Plasma viscosity or erythrocyte sedimentation rate in the diagnosis of giant cell arteritis. $\mathrm{Br}$ J Ophthalmol 1991; 75: 656-659.

16. Helfgott SM, Kieval RI. Polymyalgia rheumatica in patients with normal erythrocyte sedimentation rate. Arthritis Rheum 1996; 39: 304-307.

17. González-Gay MA, Rodríguez-Valverde V, Blanco R, Fernández-Sueiro JL, Armona J, Figueroa M et al. Polymyalgia rheumatica without significantly increased erythrocyte sedimentation rate. Arch Intern Med 1997; 157: 317-320.

18. Proven A, Gabriel SE, O'Fallon WM, Hunder GG. Polymyalgia rheumatica with low erythrocyte sedimentation rate at diagnosis. J Rheumatol 1999; 26: 1333-1337.

19. Gabriel SE, O’Fallon WM, Achkar AA, Lie AJT, Hunder GG. The use of clinical characteristics to predict the results of temporal artery biopsy among patients with suspected giant cell arteritis. J Rheumatol 1995; 22: 93-96.

20. Fauci AS. Síndromes de las vasculitis. En: Fauci AS, Braunwald E, eds. Harrison. Principios de Medicina Interna. Madrid, McGraw-Hill, 1998; 2171-2184.

21. Desmet GP, Knockaert DC, Bobbaers HJ. Temporal arteritis: the silent presentation and delay in diagnosis. J Intern Med 1990; 227: 237-240.

22. Chmelewski W, Mc Knight KM, Agudelo CA, Wise CM. Presenting features and outcomes in patients undergoing temporal artery biopsy. Arch Intern Med 1992; 150: 1690-1695.

23. Weiss LM, Gonzalez E, Miller SB, Agudelo CA. Severe anemia as the presenting manifestation of giant cell arteritis. Arthritis Rheum 1995; 38: 434-436.

24. González-Gay MA, García-Porrúa C, Llorca J, Hajeer AH, Brañas F, Dababnah A et al. Visual manifestations of giant cell arteritis. Trends and clinical spectrum in 161 patients. Medicine 2000; 79: 283-92. 